\title{
PUBLICIDAD, CONSUMO Y GOBIERNO DE LA SUBJETIVIDAD
}

\author{
Osvaldo CORRALES* \\ "La mente es la superficie de inscripción para el poder, \\ cuyo instrumento es la semiología" \\ Michel Foucault
}

Con toda seguridad la publicidad es el formato comunicativo con el que más frecuentemente tenemos que habérnoslas en nuestra vida cotidiana. En la presentación de su libro "El espectáculo del deseo: usos y formas de la persuasión publicitaria", Carlos Lomas $^{1}$ señala que a los diez años un niño o niña habrá visto unos 200 mil anuncios publicitarios sólo a través de la televisión; una cifra abrumadora, en una actividad en la que aparentemente todo aparece marcado por el sino del exceso. Sin embargo y a pesar de que su importancia no ha hecho sino acrecentarse con el desarrollo de las llamadas sociedades de consumo, para las Ciencias Sociales en general y para las disciplinas vinculadas a la Comunicación en particular, la publicidad ha sido un objeto de interés menor, una especie de epifenómeno del capitalismo, cuya función resulta tan obvia y tan transparente que apenas si ha suscitado controversia por sí misma. Ciertamente, hay toda una tradición analítica, vinculada especialmente a la semiótica, que se ha interesado por los anuncios publicitarios individuales considerándolos como piezas discursivas, pero la publicidad en tanto dispositivo y régimen general de significación y representación ha despertado relativamente poco interés teórico.

En nuestra opinión dicha "naturalización" de la función de la publicidad, observable tanto entre sus defensores como entre sus críticos, representa uno de los logros más notables de este sistema y es precisamente la que nos lleva a interesarnos por su actual estatuto en el seno de la sociedad y la cultura. La hipótesis que intentaremos justificar en este artículo es que la principal función que la publicidad estaría cumpliendo hoy en día, lejos de lo que se piensa, no sería la de convencer-seducir al público para que adquiera los productos y servicios disponibles en el mercado, sino más bien la de insertarse como un dispositivo subjetivante, es decir, como un dispositivo que genera unas ciertas condiciones de posibilidad para la constitución (o disolución) de determinados sujetos sociales mediante el establecimiento y la delimitación de un conjunto de espacios de reconocimiento e identificación que nos llevan a entendernos y relacionarnos con nosotros mismos y con los demás como si fuésemos (y fueran) sujetos de un determinado tipo, contribuyendo de este modo al desarrollo de lo que Foucault ${ }^{2}$ ha llamado el gobierno de la subjetividad.

\section{La reflexión en torno a la publicidad: de la función informativa al espectáculo del deseo}

Antes de exponer nuestro argumento se impone una breve revisión (panorámica y necesariamente incompleta) de los principales enfoques desde los que se ha conceptualizado el fenómeno de la publicidad y se ha intentado comprender la dinámica

\footnotetext{
* Profesor del Instituto de la Comunicación e Imagen. Universidad de Chile.

1 LOMAS, Carlos. El espectáculo del deseo. Usos y formas de la persuasión publicitaria. Octaedro, Barcelona. Primera edición, 1996.

2 FOUCAULT, Michel: "La gubernamentalidad". Págs.: 175 - 197. En: FOUCAULT, Michel: Estética, ética y hermenéutica. Paidós. Barcelona. Primera edición, 1999.
} 
que estructura sus efectos; esta mirada resultará de gran importancia puesto que uno de los elementos que a nuestro juicio está a la base de esta "naturalización" de la función publicitaria es que, a pesar de sus muchas y profundas diferencias, habría entre todos estos enfoques una serie de supuestos compartidos que establecen los límites de lo que ellos nos permiten pensar acerca de la de la comunicación publicitaria y que resulta necesario problematizar para constituir un modelo que pueda integrar toda la complejidad que actualmente este fenómeno incluiría.

Una primera perspectiva (que a estas alturas parece ingenua) es la que podríamos denominar informativa. De acuerdo con ella el aumento en la oferta bienes y servicios que se produce en las sociedades postindustriales ha complejizado el mercado de tal forma que es imposible que un consumidor cualquiera pueda conocer por sí mismo la variedad de productos y servicios que existen para la satisfacción de sus necesidades, sus características, ventajas comparativas, funcionamiento y precio. En este sentido, la función de la publicidad sería básicamente la de informar a los consumidores entregándoles herramientas que les permitan dar con aquellos productos que mejor se adecuan a sus necesidades específicas orientando, de esta forma, sus decisiones de consumo en diversos ámbitos. En este enfoque, el consumo aparece guiado por necesidades "objetivas" y los bienes son consumidos con arreglo a la importancia de dichas necesidades y a la cantidad de recursos disponibles para satisfacerlas: los consumidores son vistos como sujetos individuales, libres y racionales, que toman sus decisiones de consumo de acuerdo a un cálculo exacto guiado por una evaluación del tipo costo-beneficio, es el reino del homo economicus weberiano.

Una segunda perspectiva la constituye aquella que se ha desarrollado de la mano de la psicología y, particularmente, de corrientes como el psicoanálisis. En este enfoque se abandona la idea de sujeto racional y se pone particular énfasis en las dimensiones inconscientes e irracionales de las necesidades y motivaciones que estarían a la base del consumo; los objetos ya no aparecen reducidos a su valor de uso sino que se reconoce en ellos un cierto valor de signo, una cierta capacidad de simbolización que les permite representar cosas que se encuentran más allá de su razón práctica y que rompen la asociación consumo-necesidad reemplazándola por la de consumo-deseo. La principal función de la publicidad, entonces, no es ya la de informar acerca de las características objetivas de los bienes y servicios sino más bien la de ser capaz de construir en torno a ellos (y sobre ellos) conjuntos de significados y simbolizaciones que los doten de una cierta "plusvalía de sentido" que convierta a los objetos en "objetos de deseo"”.

Una tercera perspectiva, asociada estrechamente con la anterior, la representan aquellas teorizaciones surgidas al alero de disciplinas como la sociología y la antropología que entienden al consumo básicamente como una forma simbólica de conservación y reproducción de la sociedad. Desde este punto de vista, el consumo no sólo satisface necesidades materiales sino que tiene una importante función cultural en tanto legitima y consolida formas concretas de entender la vida cotidiana en el seno de una determinada sociedad ${ }^{4}$. Por supuesto que, en este contexto, se considera que la publicidad es uno de los dispositivos más importantes de producción de sentidos sociales dentro de las sociedades contemporáneas, en tanto es en ella en donde radica la función de sobresignificar los

3 GONZÁLEZ REQUENA, Jesús y ORTÍZ DE ZÁRATE, Amaya. El espot publicitario. La metamorfosis del deseo. Cátedra, Madrid. Primera edición, 1995.

4 BAUDRILLARD, Jean. El sistema de objetos. Siglo XXI, México. 1969. 
objetos cristalizando mediante su discurso insistente y repetitivo los modos, estilos y gustos de las clases dominantes, que quedan designados como "distinguidos" en contraposición con otras formas de consumo que caen en la categoría de "corrientes" o "vulgares": no obstante ser un discurso que habla indiscriminadamente, la publicidad permite discriminar los distintos tipos de consumo ubicando a cada quien en su sitio dentro del orden social. Observamos que en este caso la óptica desde la que se analiza la función de la publicidad se amplía de modo tal que ésta ya no le habla a un sujeto individual sino a uno colectivo y lo hace desde los valores e intereses de quienes detentan (y ostentan) la hegemonía sobre el orden social: el discurso publicitario es el discurso del poder y el orden cultural.

La perspectiva "psicológica" y la "socio-antropológica" no son contradictorias ni discontinuas; de hecho, la denominada "Teoría Crítica" ha devenido como una suerte de síntesis entre ambas desarrollando una perspectiva de orden freudo-marxista. De acuerdo a la lectura de autores como Adorno, Horkheimer o Marcuse, el consumo y disfrute de objetos y mercancías actuaría como un sistema de gratificaciones que colmaría (espuria e ilusoriamente) el sentido de vacío existencial y frustración de los individuos frente a una sociedad alienante e injusta. El consumo representaría una forma consentimiento y acuerdo con el orden establecido y con las relaciones de poder y propiedad que se encuentran vigentes en su interior, al situar la vida de las personas al servicio de la conservación del orden productivo. La publicidad pasa a ser concebida como una herramienta de control y manipulación social que, por medio de la enajenación de sus deseos y su alienación ideológica, tendría como objetivo el adoctrinamiento y normalización de los sujetos en las coordenadas de los valores de la sociedad de clases.

\section{El problema del sujeto: pasividad y esencialismo}

Como resulta fácil advertir, la imagen dominante del público en las distintas aproximaciones al tema de la publicidad descritas en el apartado anterior (excepción hecha de la perspectiva económica a la que hemos denominado "ingenua" dado su marcado sesgo racionalista) es la de un sujeto pasivo que se encuentra inerme frente a la influencia de los mensajes publicitarios, los que tienen la capacidad de manejar sus emociones, influir sobre sus instintos, "inventarle" necesidades y, en fin, conducirlo a hacer cosas que, al parecer, de otro modo no haría.

Frente a esa visión "pasiva" del público es que van a reaccionar una serie de enfoques que centrarán sus análisis en las posibilidades de los receptores frente a los mensajes que se les envían. Es el caso de la llamada teoría de los Usos y Gratificaciones y muy especialmente del movimiento iniciado por los Cultural Studies; en ambos casos el interés se desplazará desde conocer qué hacen los mensajes de los medios de comunicación (entre ellos la publicidad) con las personas a saber qué hacen las personas con dichos mensajes. En este sentido, los estudios desarrollados en el marco de los Cultural Studies resultarán de gran interés dado que, sin abandonar una visión crítica del proceso, pondrán en jaque el pesimismo frankfurtiano al evidenciar las posibilidades de recepción crítica que poseen quienes son destinatarios de los mensajes producidos desde los medios.

Traduciendo la perspectiva de este enfoque a nuestra problemática, si bien los anuncios no resultarían completamente inofensivos, los individuos tampoco se encontrarían completamente indefensos frente a ellos: el diseño de cada anuncio contendría dentro de sí

5 BOURDIEU, Pierre. La distinción. Criterios y bases sociales del gusto. Taurus, Madrid.1988. 
una "lectura preferente" que se correspondería con el sentido que ha querido otorgarle quien lo ha elaborado; sin embargo, en función de su experiencia común (dentro de la cual juega un rol de primer orden, aunque no exclusivo, la variable clase social) los distintos públicos desarrollarían sus propios códigos que no necesariamente se corresponden con los de la fuente y en función de los cuales podrían realizar sus propias interpretaciones de dichos mensajes, reapropiándose creativamente de sus contenidos y llegando incluso a desarrollar lecturas que pueden resultar completamente opuestas a los intereses originales de quien los ha creado (e incluso del orden que intentan fomentar), con lo que la unidad monolítica del sistema se rompe por el lado de los receptores y reaparece, aunque con timidez, la posibilidad de cambio y transformación que era negada por el fatalismo de la Teoría Crítica.

Con todo lo importante que ha sido el debate en torno a la actividad de los receptores y los aportes que ha significado en términos de permitir el desarrollo de una visión cada vez más compleja del proceso comunicativo, bajo estas diferencias y, por lo tanto, en un nivel más profundo de aquél donde ellas son posibles y resultan relevantes, podemos observar que existe una serie de asunciones con relación a lo que los sujetos son y a la forma en que llegan a ser eso que son, que todos los enfoques hasta aquí revisados dan por descontadas y que representan la base común desde la que articulan sus posiciones teóricas.

Dichas asunciones compartidas tienen que ver con una noción esencialista del sujeto, es decir, con la idea de que existe un sujeto psicológico dado, universal, pre cultural y pre - linguiístico, originado en una serie de estructuras psicológicas profundas y cuyos bloques constitutivos serían la racionalidad, el autocontrol, la autonomía y la independencia. Este sujeto psicológico (núcleo constitutivo de una naturaleza humana invariable) sería integrado a la sociedad a través de la acción de una serie de agentes de socialización, tales como a familia, la escuela o los propios medios de comunicación, todos los cuales desarrollarían esfuerzos tendientes a inculcar ciertas formas culturalmente específicas de entender, interpretar y actuar en el mundo.

En otro lugar ${ }^{6}$ hemos especificado cuáles son las señas específicas que permiten afirmar esto respecto de las principales teorías que han sido elaboradas para comprender la dinámica y efectos de los medios en la sociedad; queremos centrarnos aquí sólo en el tema de la publicidad y señalar que este supuesto tiene importantes consecuencias en la forma en que concebimos sus funciones y efectos puesto que, si existe un sujeto psicológico universal, pre - cultural y anterior a cualquier experiencia comunicativa, entonces los mensajes de los medios de comunicación -y, especialmente de la publicidad como su formato más paradigmático-, sólo podrán afectar externamente las estructuras fundamentales de ese "yo" (para bien o para mal, dependiendo del caso), teniendo la capacidad de influirlas pero en ningún caso de constituirlas. El esencialismo supone que las estructuras internas del sujeto se mantienen relativamente invariables en su definición (aunque más o menos robustas en sus posibilidades) y que la publicidad sólo puede movilizarlas pero no modificarlas ni menos aún constituirlas, lo cual resulta contradictorio tanto con una serie de evidencias que cuestionan esa inmutabilidad y universalidad como con los objetivos que la publicidad se fija, con no poco éxito, en relación con el público, y

6 CORRALES, Osvaldo. La publicidad en el proceso de producción política de sujetos. Una revisión desde la psicología crítica y la comunicación. Tesis para opta al grado de magíster. Universidad de Chile. Santiago, 2005. 
que se revelan con especial fuerza en la propia emergencia del relativamente nuevo, e históricamente inédito, tipo de sujeto que es el consumidor.

\section{El giro interpelativo: la publicidad y la producción de sujetos}

Resituar el estatuto y funciones de la publicidad en un esfuerzo por explicar cómo y por qué es que ha llegado a ocupar un lugar tan prominente en el marco de las sociedades postindustriales, supone en primer término observar la forma en que ésta ha ido mutando y se ha ido posicionando a través de las distintas transformaciones del propio capitalismo.

De acuerdo a lo planteado por el sociólogo español Jesús Ibáñez ${ }^{7}$, una de las principales transformaciones experimentadas por la publicidad en la transición desde el capitalismo de producción al capitalismo de consumo (las dos grandes etapas de su desarrollo), es que ésta pasa de estar centrada en la información a estar centrada en la expresión: no buscará más dar cuenta de las características "objetivas" de los productos sino que intentará dotarlos de una significación específica que sea coherente con la imagen que se pretende dar a la marca a la que pertenecen, y mediante la cual se transformen en signos de aquello que la publicidad afirma. En esta etapa, por lo tanto, la publicidad deja de ser referencial (informativa) y se vuelve estructural porque pasa a formar parte de la estructura de producción del producto como signo (su materialidad no tiene apenas importancia) y de su necesidad simbólica. Este movimiento supone, entre otras cosas, que la publicidad, en tanto modalidad enunciativa, deja de centrarse en los objetos que anuncia para pasar a concentrarse en los sujetos a los que se dirige: deja de informar sobre los productos para enfocarse de lleno en el individuo, buscando darle forma y fijarlo (sujetarlo) en una determinada posición: la interpelación, en el sentido que Athusser da al término ${ }^{8}$, es el mecanismo por medio del cual la publicidad logrará este propósito, generando una posición en la que el individuo pueda reconocerse y en torno a la cual pueda articular su subjetividad y constituirse en un sujeto de un determinado tipo. Lo que se consuma entonces es un "giro interpelativo" mediante el cual la publicidad pasa de lleno a participar en el proceso de producción de sujetos.

El anterior argumento resulta en apariencia similar al desarrollado por la Screen Theory en relación con los filmes de Hollywood pero, en realidad, nuestra concepción del proceso es muy diferente. En primer lugar, porque a diferencia de lo postulado por la Screen Theory (en uno de los puntos que más críticas le ha valido), no consideramos que los textos publicitarios (ni de ningún otro tipo) determinen de un modo absoluto al sujeto, conduciéndolo de un modo irremediable a constituirse como tal de una forma específica; creemos, más bien, que la publicidad opera generando posiciones de sujeto (que dado el carácter polisémico de los textos serán multiformes), que son lugares desde los cuales es posible hablar y relacionarse con uno mismo y con los demás de una determinada forma y que pueden ser ocupadas, o no, por aquellos a quienes se dirige la interpelación. Pero, en segundo lugar y como ya adelantáramos, partimos también de una forma muy distinta de conceptualizar la subjetividad y la identidad que está en línea con lo postulado por el

\footnotetext{
7 IBÁÑEZ, Jesús: "Una publicidad que se anuncia a sí misma”. Págs.: 117 - 123. En: Revista Telos. NN $^{\circ}$. Madrid, 1987; IBÁÑEZ, Jesús: "La publicidad, un remedio contra el pánico". Págs.: 31 - 38. En: BOTANA, Ana (ed.): Publicidad, semiótica e ideología. Cuadernos contrapunto. Editorial Contrapunto. Madrid. 1990.

${ }^{8}$ Especialmente en su trabajo "Ideología y aparatos ideológicos de Estado" (Nueva Visión, Buenos Aires. 1974.)
} 
construccionismo y el post - estructuralismo y en función de la cual no las consideramos como entidades coherentes, plenamente integradas, estables y claramente delimitadas, que pueden ser definidas y determinadas de una vez y para siempre o por largos períodos de tiempo; más bien, las concebimos como fragmentarias, difusas, inestables y contradictorias, de modo tal que las posiciones de sujeto (como las propuestas por la publicidad) no aparecen como lugares definitivos de fijación de la identidad o la subjetividad sino sólo como puntos provisionales de articulación a los que se recurre en el marco de interacciones locales específicas en perspectiva al desarrollo de determinadas acciones. Esto quiere decir, entre otras cosas, que no porque una determinada posición de sujeto no haya sido ocupada en un momento cualquiera no vaya a serlo en otro, lo cual nos conduce directamente al tercer punto que tiene que ver con una forma distinta de concebir los procesos de lectura e interpretación y su relación con la acción social. En efecto, desde nuestra perspectiva, el desarrollo de ciertos tipos de interpretación (y la ocupación, en función de ellos, de determinadas posiciones de sujeto) no debe ser analizado única ni prioritariamente en función de los "recursos culturales" o la "capacidad crítica" de que, supuestamente, esté dotado el individuo (como ocurre, por ejemplo, en el caso de los Cultural Studies), sino que debe considerarse, más bien, desde el punto de vista de las justificaciones que genera, las acciones que posibilita y el tipo de objetivos que permite alcanzar en el marco de relaciones e interacciones concretas, en un curso de vida específico9; las interpretaciones no deben ser consideradas, simplemente, como abstracciones conceptuales individuales (como representaciones mentales privadas) sino también, y ante todo, como repertorios o recursos para la acción social, que generan condiciones de posibilidad para que nos relacionemos con nosotros mismos y con los otros como si fuésemos personas de un determinado tipo, y que son útiles en el marco de determinadas relaciones e interacciones.

\section{Publicidad y gobierno de la subjetividad}

El hecho de que abandonemos la imagen de un "yo" integrado, coherente y plenamente integrado y reconozcamos la existencia de grados mayores o menores de dispersión, fragmentación e inestabilidad no significa que, en lo que respecta a la constitución de los sujetos, la publicidad (como dispositivo general) no produzca una cierta normatividad. Cuando la publicidad nos interpela de la forma que sea, sus mensajes llevan implícitas una serie de suposiciones en relación con nuestra condición: por ejemplo y a un nivel muy elemental, se parte de la base que somos autónomos y libres para decidir; pero no sólo eso, también se asume que tenemos la capacidad real de elegir, es decir, que estamos habilitados materialmente (económicamente) para el consumo. Esta suposición, que aparece naturalizada pero que en realidad es gratuita, esta en el origen mismo de todo un régimen normativo construido a través del discurso publicitario, y esto por dos razones: por una parte, porque en función de ella se organiza toda una jerarquía y aparece todo un universo de exclusiones parciales o totales, materiales pero también simbólicas (nadie está habilitado para realizar todos los consumos en todos los momentos, la capacidad de consumir es siempre finita $\mathrm{y}$, además, existen impugnaciones de diverso tipo); por otra, porque la publicidad es, casi por definición, un habla indiscriminada: puede tener un público objetivo, pero sus mensajes están disponibles para todos, y no sólo eso, casi

9 BLACKMAN, Lisa y WALKERDINE, Valerie. Mass hysteria: critical psychology and media studies. Palgrave, London. 2001 
siempre se imponen a nuestra mirada aunque no los busquemos por lo que, en todo momento, nos recuerda cuáles son los ámbitos de los que participamos y de cuáles somos sistemáticamente excluidos; sabemos quienes son "otros" respecto de nosotros, pero también conocemos para quienes nosotros constituimos una oscura "otredad".

La cuestión del otro es un elemento clave en nuestra constitución como sujetos: somos capaces de relacionarnos con nosotros mismos como si fuésemos personas de un determinado tipo en tanto existen otros respecto de los cuales nos diferenciamos y que, a través de su diferencia, confirman nuestra identidad. Ahora bien, desde el punto de vista de la construcción de una normatividad cualquiera esa "otredad" (lo otro de la norma, lo anormal) nunca es codificada como mera diferencia sino que siempre es connotada negativamente, presentándose como carencia o incapacidad; siempre aparece patologizada de alguna forma pasando a representar todo aquello que tememos o detestamos y, en relación con dicha imagen, la propia normatividad aparece siempre construida como natural y autoevidente. En nuestra opinión, desde los supuestos de habilitación para el consumo la publicidad genera precisamente una normatividad de este tipo: la "otredad" del consumo se transforma en aquello que tememos, y articulamos nuestras luchas y padeceres cotidianos para evitar caer allí, puesto que esa condición representa la incompetencia y el fracaso; ser los "otros" absolutos del consumo, aquellos que ni siquiera pueden hipotecar su futuro en el presente para participar de sus intercambios rituales, supone ser excluido del espacio social y verse condenado al ostracismo. Es esta propia "otredad" la que permite y posibilita la naturalización de ese modelo normativo: el hecho de que haya quienes fracasan, demuestra que nuestro éxito es merecido, que lo obtenemos gracias a nuestro esfuerzo, por lo que el orden social deviene como justo. Por medio de la publicidad, por tanto, no sólo se generan y promueven posiciones de sujeto, también se rechazan, desprecian, patologizan y desnaturalizan otras, de forma tal que se producen condiciones de posibilidad que favorecen la aparición de determinadas formas de subjetividad: deseamos vivamente ser sujetos de un determinado tipo y estamos dispuestos a sacrificarnos por ello.

Dichas condiciones de posibilidad aparecen reforzadas por toda una red de prácticas e instituciones mutuamente solidarias que contribuyen a generar un tipo de gubernamentalidad que es tanto el resultado de la racionalidad política imperante como de las tecnologías del yo al uso ${ }^{10}$, y que más que asociarse con la concentración del poder en determinadas esferas se vincula con su circulación, distribución y ejercicio en distintos niveles y de diversas formas en ese nuevo tipo de configuración societal que ha llegado a ser conocido como sociedad de consumo, con sus habitantes acreditados, los consumidores.

10 FOUCAULT, Michel. Tecnologías del yo y otros textos afines. Paidós, Barcelona. Primera edición, tercera reimpresión, 1996. 


\section{Referencias bibliográficas}

ALTHUSSER, Louis. Ideología y aparatos ideológicos de estado. Nueva Visión, Buenos Aires. 1974.

BAUDRILLARD, Jean. El sistema de objetos. Siglo XXI, México. 1969.

BLACKMAN, Lisa y WALKERDINE, Valerie. Mass hysteria: critical psychology and media studies. Palgrave, London. 2001

BOURDIEU, Pierre. La distinción. Criterios y bases sociales del gusto. Taurus, Madrid.1988.

CORRALES, Osvaldo. La publicidad en el proceso de producción política de sujetos. Una revisión desde la psicología crítica y la comunicación. Tesis para opta al grado de magíster. Universidad de Chile. Santiago, 2005.

FOUCAULT, Michel: “La gubernamentalidad”. En: FOUCAULT, Michel: Estética, ética y hermenéutica. Paidós. Barcelona. Primera edición, 1999. Págs.: 175 - 197.

FOUCAULT, Michel. Tecnologías del yo y otros textos afines. Paidós, Barcelona. Primera edición, tercera reimpresión, 1996.

GONZÁLEZ REQUENA, Jesús y ORTÍZ DE ZÁRATE, Amaya. El espot publicitario. La metamorfosis del deseo. Cátedra, Madrid. Primera edición, 1995.

IBÁÑEZ, Jesús: "Una publicidad que se anuncia a sí misma”. En: Revista Telos. № 8. Madrid, 1987. Págs.: $117-123$.

IBÁÑEZ, Jesús: "La publicidad, un remedio contra el pánico". En: BOTANA, Ana (ed.): Publicidad, semiótica e ideología. Cuadernos contrapunto. Editorial Contrapunto. Madrid. 1990. Págs.: 31 - 38.

LOMAS, Carlos. El espectáculo del deseo. Usos y formas de la persuasión publicitaria. Octaedro, Barcelona. Primera edición, 1996. 